\title{
Review on Utilizing Grp78 on Endometrial Cancer Therapy
}

\author{
Yunqi Bao ${ }^{1, *}$ \\ ${ }^{1}$ Land and Food System, University of British Columbia, Vancouver, BC, Canada, V6T 1Z1 \\ *Corresponding author. Email: baoyunqinanjing@163.com
}

\begin{abstract}
The significance of Grp78 gene expression in cancer has been confirmed for many years, yet the feasibility of applying Grp78 targeting treatment in endometrial cancer remains questioned. By reviewing past research that specifies the therapeutic value of Grp78, this article concludes that Grp78 has the potential of acting as a prognostic factor, and target for promoting chemosensitivity and radiosensitivity in endometrial cancer treatment. This article also includes the mechanism behind Grp78 expression-induced chemoresistance, which provides an alternative therapy for drug resistance. Despite various applications exist, huge risks come with manipulating ER stress-related genes, which requires further study.
\end{abstract}

Keywords: Endometrial cancer, Grp78, ER stress, Chemoresistance, Radioresistance

\section{INTRODUCTION}

Endometrial cancer (EC) has been the fifth most common cancer in women, which covers $4.8 \%$ of female cancer patients worldwide[1]. Viewing from historical data, the annual age-standardized incidence rate (ASR) in 26 out of 43 countries exhibits a significant increase from 2001 to 2010 [2]. Data estimated in United State showed that 60,050 new cases were diagnosed in 2016, and 10,470 cases caused death[3]. The United States also has the highest ASR of 19.1/100000 estimated in 2012, and this number is projected to reach $42.13 / 100000$ by $2030[1]$. The latest statistic estimates 65,620 new cases occurred in the USA in 2020, among which 12590 cases caused death. There has been a steady increase of $1.3 \%$ per year from 2007-2016 in EC incidence, which is possibly affected by the increasing obesity rate among women[4]. All these data clearly demonstrate great demand for treatment against EC. Several past pieces of research have revealed that Grp78 has a huge application prospect as a precise disease indicator as well as a new therapeutic target for EC patients. This article aims to review the correlation between Grp78 and EC, and some potential applications on disease treatments or diagnosis. By summarizing research on Grp78 in EC so far, it would be easier to identify existing obstacles to improving patients' survival. Also by relating current EC specialized treatment with treatments applied in other cancer types, it is possible to apply alternative therapies against drug resistance.

\section{INTRODUCTION ON GRP78 IN EC}

Grp78 has been long known as the biomarker for endoplasmic reticulum (ER) stress, and it has been widely used in many cancers as an indicator for activation of ER stress and unfolded protein response (UPR) [5]. Scientists have already found that there is a general correlation between UPR and tumor cell proliferation in almost all types of cancer, factors involved in ER stressUPR pathway are therefore becoming a popular therapeutic target.

Although ER stress has been proved to be active in many other cancers, its activity in EC was first verified in 2012 by Bifulco et.al. [6]. During the research, 12 EC tissue were collected from abdominal hysterectomy on patients. Several examinations and analyses were performed on samples, including total RNA extraction and Real-Time Reverse Transcription-PCR, Immunohistochemistry with anti-Grp78 peptide antibody, and Western blot. The result shows that both RNA expression level and protein level of some unfolded protein response (UPR) markers, including Grp78, CHOP, and ATF6, are significantly higher in endometrial tumor cells than in normal cell. This result verified that ER stress is activated in EC. It is also found that inhibiting Grp78 protein expression using short hairpin RNA would 
downregulate cell growth and proliferation of Ishikawa cells. This is further confirmed in 2014, where AN3CA cells treated with Grp78-binding-antibody have lower cell viability [7]. Migration and invasion ability are also suppressed without Grp78 expression, suggesting that Grp78 may be a positive regulator for tumor cell migration.

With the fact that ER stress is activated in EC and correlate with cell growth, it is possible to discover signals along its pathway as potential therapeutic targets. Further studies indicate that Grp78 as a key indicator of ER stress can also impact patients' sensitivity towards medication, or act as an indicator of patients' survival.

\section{GRP78 AS A PROGNOSTIC FACTOR}

Grp78 is a crucial factor involved in ER stress and UPR. Its expression level in visceral adipocytes predicts disease progression and patient survival in EC [8]. In this research in 2014, $246 \mathrm{EC}$ patients were recruited for data collection, among which 179 EC patients had their

A
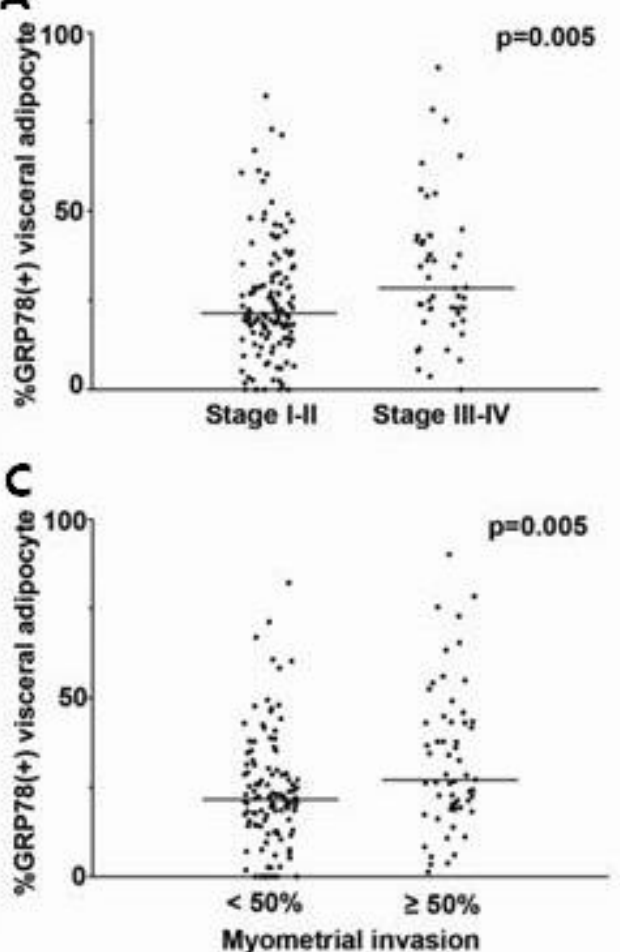

visceral adipocytes analyzed for Grp78 and $\mathrm{CHOP}$ expression level, and also had their disease stage, disease progression, and disease-free survival (DFS) evaluated. Among 179 patients, $95 \%$ of them have Grp78 expression detected in their visceral adipocytes, and the most common GRP78-positive-adipocytes per individual sample is $24 \%$. Through statistical assessment with multi characteristics, higher GRP78-positive-adipocyte is strongly correlated to advanced-stage disease, deeper myometrial invasion (Figure 1), and worse 5-year disease-free survival (Figure 2). These results suggest that visceral adipocytes Grp78 expression, which has never been used as a prognostic predictor before, can reflect advanced stage, deep myometrial invasion, and overall survival. This is confirmed again in 2018 with data collected from 130 endometrial carcinoma cases, where Grp78 expression is positively correlated with FIGO stage, pathological type of adenocarcinoma, histological grade, lymph node metastasis, and overall survival [9]. Although myometrial invasion is found not related to Grp78 expression in this study, Grp78 still has the potential to be an independent prognostic factor.
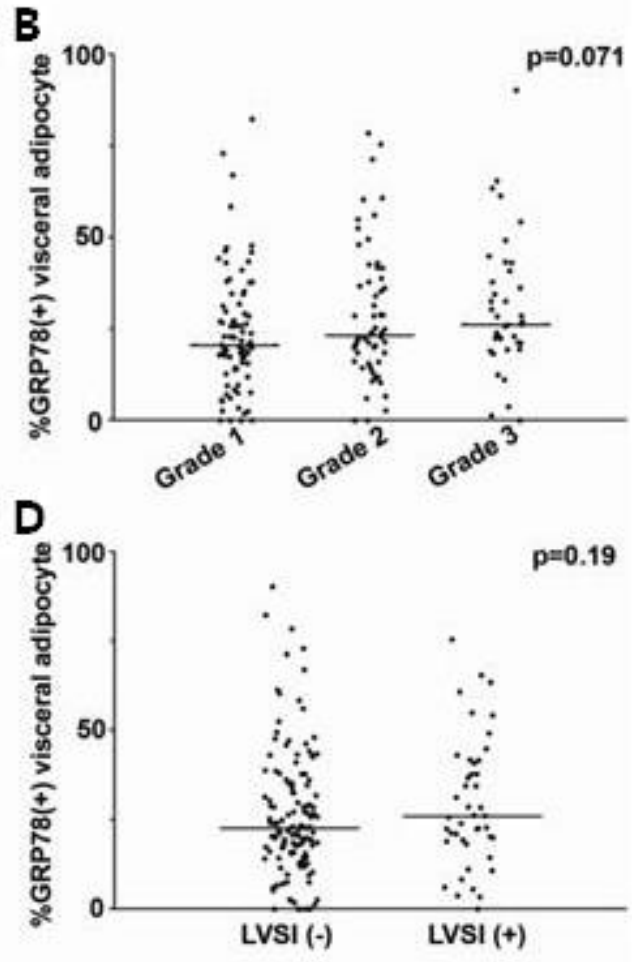

Figure 1. Proportion of GRP78 (+) expression in visceral adipocytes based on FIGO stage (A), FIGO grade (B), deep myometrium invasion (C), and LVSI $(+)(D)[8]$. 


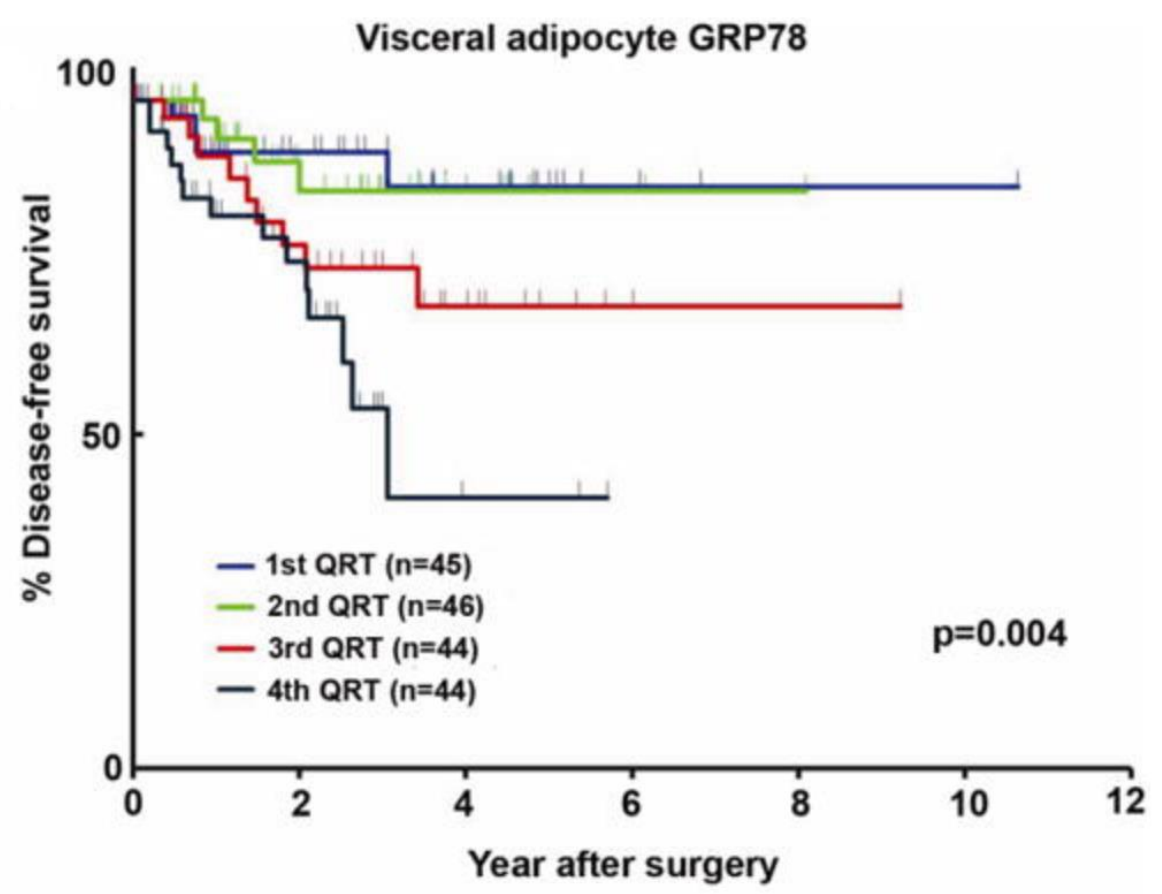

Figure 2. 5-year disease-free survival of patients with different quartiles of Grp78 expression in visceral adipocyte [8].

\section{GRP78 INDUCED RADIATION RESISTANCE}

Grp78 was found to be the mediator that causes breast cancer-initiating cells (CICs) to have radiation resistance [10]. As background information, CICs were defined as "a relatively radioresistant subpopulation of breast cancer cells and increase in numbers after short courses of fractionated irradiation" in 2006 by Tiffany et.al. [11]. In the same paper, CICs were found to gain radioresistance because of decreased ROS induction and activation of the Motch-1 pathway after radiation, and they are the main accelerator of tumor cell proliferation during radiation therapy treatment gaps.

According to later research done in 2013, CICs isolated from breast cancer cell line exhibits higher selfrenewal ability, resistance to irradiation and Grp78 gene expression as compared to normal tumor cells [10]. To verify the necessity of Grp78 in radioresistance induction, Grp78 in CICs are overexpressed and silenced by pcDNA3.1(+)/hGRP78 and pSuper/GRP78 RNAi transformation respectively. Grp78 mRNA level shows a significant increase and decrease in two transfected cells as compared to untreated CICs (Figure 3a,3b). Transfected cells, as well as normal tumor cells and untreated $\mathrm{CICs}$, are then exposed to radiation to evaluate their survival curves. The result (Figure 3c,3d) shows survivorship significantly decreases when Grp78 expression is suppressed. Also, survivorship increases with enhancement in Grp78 expression in a dosedependent manner. In other words, radiation therapy is more effective when the Grp78 gene is silenced, indicating that Grp78 is a potential therapeutic target against radioresistance. 

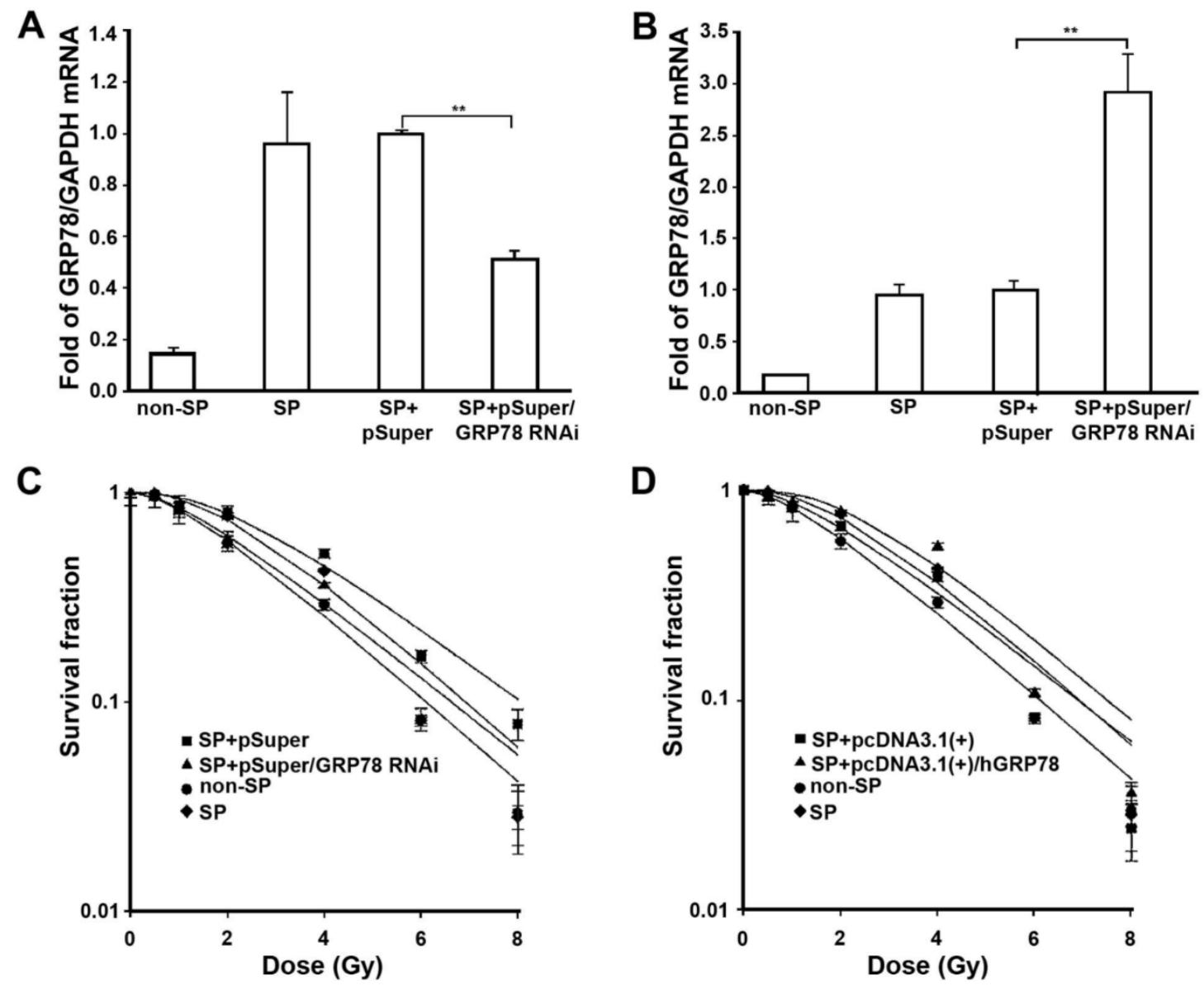

Figure 3. Reduction of Grp78 expression level in SP cells mediated by pSuper/GRP78 RNAi (A). Increase of Grp78 expression level in SP cells mediated by pcDNA3.1(+)/hGRP78 (B). SP cells treated with pSuper/GRP78 RNAi shows increase in sensitivity towards radiation (C). SP cells treated with pcDNA3.1(+)/hGRP78 is less affected by ionizing radiation [10].

\section{GRP78 INDUCED CHEMOTHERAPY RESISTANCE}

Grp78 was found to be the key factor that causes resistance against paclitaxel and cisplatin in EC cells [12].

Since the combination of cisplatin and paclitaxel remains one of the most effective chemotherapies for EC, developed chemoresistance against these two medicines largely reduces the patient's survivorship. Study in 2012 suspects that Grp78 plays a crucial role in this process. To verify the impact of chemotherapy drugs on normal tumor cells, researchers took Ishikawa cells and HHUA cells and treated them with a chemotherapy drug. Cell viability of Ishikawa cells is reduced to $62 \%$ and $67 \%$ by paclitaxel and cisplatin respectively. Cell viability of HHUA cells is reduced to $54 \%$ and $82 \%$ by paclitaxel and cisplatin respectively. To verify the involvement of Grp78 in chemoresistance, some Ishikawa cells and HHUA cells are pre-treated with estrogen, which is a proliferative factor that increases cell viability. These two groups are then transfected by siGrp78 and siCont, and the siGrp78 transfected group shows an $80 \%$ lower cell viability as compared to siCont transfected group (Figure 4). It is clear that the estrogen effect is counteracted by knocking down Grp78 expression. A reduction in cell viability is also observed when untreated Ishikawa cell and HHUA cell are transfected with siGrp78 and then exposed to paclitaxel and cisplatin. To be more specific, $13 \%-15 \%$ reduction is found in paclitaxel treatment, and $8 \%-12 \%$ reduction is found in cisplatin treatment. These results indicate that chemosensitivity is increased by inhibiting Grp78 expression. To further confirm the correlation between decreased Grp78 expression and increased cell apoptosis, the researcher looked at some factors involved in the Grp78-apoptosis pathway. As the result, cleaved caspase- 3 and PARP are detected by Western blotting, demonstrate that upregulated Grp78 level is the cause of increased chemosensitivity. 

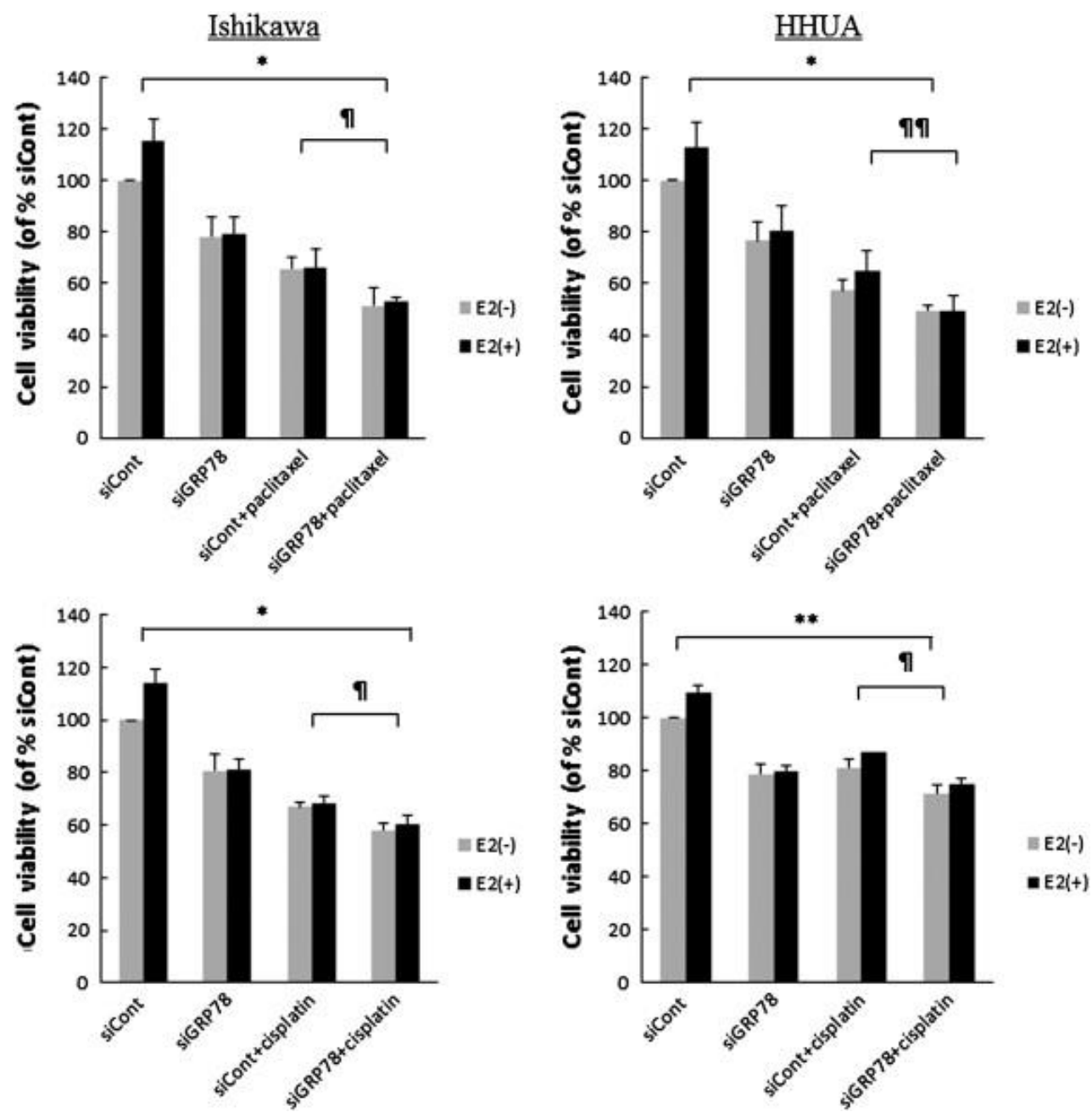

Figure 4. Change in sensitivity toward paclitaxel and cisplatin of Ishikawa and HHUA cell line under inhibition of Grp78 expression [12].

One mechanism behind Grp78 expression causing chemoresistance involves a regulator called ATK, which can be induced by chemotherapy treatment, hence lead to subsequent overexpression of Grp78 [13].

According to Gray et.al. in 2013, Grp78 acts as both downstream and upstream regulator of ATK and forms a positive feedback loop. Researchers first verified that cisplatin induces a higher Grp78 expression in various EC cell lines, and the knockdown of Grp78 would result in higher apoptosis under cisplatin treatment. Researchers then studied the regulatory relationship between Grp78 and ATK. In Ishikawa cells treated with cisplatin, ATK is found to be activated at 24 hours and 48 hours after treatment, followed by induction of Grp78 expression after 48 hours of treatment. In Ishikawa cells treated with an ATK inhibitor (MK2206), there is a significant reduction in phosphorylated ATK level, as well as $>85 \%$ reduction in basal Grp78 expression level. When cells having their ATK blocked are treated with cisplatin, no significant Grp78 expression is observed, suggesting that ATK is an upstream regulator of Grp78. Interestingly, knockdown of Grp 78 by siRNA in Ishikawa cells would result in a modest reduction in basal activated
ATK level. Furthermore, when these cells are treated with cisplatin, no increase in ATK activity is observed during 72 hours period after treatment. This result suggests that Grp78 contributes to ATK activation under cisplatin treatment. However, when knockdown AN3CA cells are treated with cisplatin, a reduced yet low level of ATK activation is observed, suggesting that Grp78 is not a requirement for ATK activation in response to cisplatin. Nevertheless, researchers confirm that in both AN3CA and Ishikawa cell lines, above a certain concentration of ATK inhibitor would sensitize tumor cells to cisplatin regardless of Grp78 siRNA presence. In other words, by inhibiting ATK alone, chemoresistance towards cisplatin can be suppressed through a subsequent reduction in Grp78 expression. Another ATK relative research in 2013 found that the constitutive upregulation of a kinase called serum-and glucocorticoid-regulated kinase 1 (SGK1) would induce resistance to selective pharmacological inhibition of ATK, which is involved in PI3K/ATK pathway deregulation in most EC patients [14]. SGK1 is a serine/threonine-protein kinase that is similar to the ATK family of kinase in function and structure. It normally acts as a less important growth factor in healthy cells, as the knockdown of SGK1 gene seems to have a 
mild effect on hair growth [15]. Previous research also found that SGK1 expression can be suppressed by DTTinduced-ER stress in a dose-dependent manner, but the mechanism behind this regulation is still unclear [16]. While in tumor cells, SGK1 can be an alternative effector that functions downstream of PI3K signaling and hence induces resistance to cell apoptosis. An inhibitor of SGK1 called SI113 is therefore found to reduce cell viability in glioblastoma cells in 2016 [17]. The SI113-dependent SGK1 inhibition could prevent cell restoring from radiation by activating cytotoxic autophagy, during which radiation-dependent Grp78 induction is found also inhibited. This suggests that SI113 might enhance radiosensitivity through inhibiting ER stress-related pathways, specifically through Grp78 inhibition. However, this experiment was done on glioblastoma cells and hasn't been repeated on EC cells yet. SGK1 is likely to be an upstream regulator of ATK, therefore can be an alternative target for Grp78 induced radioresistance.

\section{DISCUSSION}

From 2009 to 2015, the Five-Year Relative Survival Rates of EC in the USA was $81 \%$, with a huge difference between white and black people ( $83 \%$ vs. $62 \%$ ) [4]. The main difficulty comes from a deficiency in treatments for patients with recurrent and metastatic disease. Therefore, finding new targets is critical to extending patients' lifespan. The discovery of the important role played by Grp78 in cancer development and its various clinical value provides clues for potential new targets. Apart from having the intrinsic value as a target, the fact that Grp78 is overexpressed in EC tumor cells reveals that some ER stress-related clinical medication that works on other cancers might also be effective on EC. For example, new research in 2021 shows that 3,3'-diindolylmethane (DIM) can regulate ER stress pathway by increasing the expression of activated caspase-3, PARP, caspase-9, and $\mathrm{Bax}$, also decreasing $\mathrm{Bcl} 2$ in hepatocellular carcinoma cells [18]. DIM treatment also induces overproduction of ROS and depletes mitochondrial membrane potential, which will further induce intrinsic apoptosis.

As signaling factors involved in ER stress pathway often regulate tumor cell survival, development, and invasion, inducing apoptosis and inflammation in tumor cells via ER stress pathway is becoming a hotspot in oncotherapy [19]. There are existing targets include XBP1，JNK, eIF2a, Bcl-2 family proteins, $\mathrm{Ca} 2+$ regulated signaling pathway, etc. Some anticancer therapy mediating autophagy includes cannabinoids, photodynamic therapy, Bortezomib, and proteasome inhibitors. Despite the future of targeting ER stress signaling pathway seems bright, there is still a long way to go before matured medication applying to patients [20]. One big risk is that ER stress is often referred to as a double-edged sword because the pathways targeted by many therapies mentioned above are the exact pathway that tumor cells modified to protect themselves. This means therapy involves mediating ER stress may end up protecting tumor cells from anoxia, nutrient depletion, and immune system attack, hence promoting tumor growth. Another problem comes from the complexity of ER stress signaling pathway. Because the signaling pathway is a complicated network that humans haven't fully understood, drug resistance is always a problem as there are so many alternative pathways.

\section{CONCLUSION}

To summarize, this paper looked at the current position of Grp78 in EC treatment. Clinically, Grp78 can be taken as an indicator for the presence of ER stress and a predictor for prognosis. It is also involved in many pathways that promote tumor cells growth and induce tumor cells to gain chemoresistance and radioresistance, therefore it can be a therapeutic target to maximize outcome from existing treatments. As Grp78 is closely linked to ER stress, its significance in EC brings attention to other potentially targetable pathways that involve ER stress and improve tumor cell survivorship. These typically provide methods to manipulate ER stressinduced apoptosis and inflammation. Some of these pathways have already been studied in other cancers and have matured therapy targeting them, but yet not studied with EC patients. It is very hard to cure cancer because it is capable of migration and recurrence, and gaining resistance against treatments is almost guaranteed. The development of a new drug targeting Grp78 is not the solution to cancer but provides an alternative method that can extend patients' life.

\section{REFERENCES}

[1]Morice, P., Leary, A., Creutzberg, C., Abu-Rustum, N., \& Darai, E. (2016) Endometrial cancer. The Lancet, 387(10023), 1094-1108.

[2]Joannie Lortet-Tieulent, Jacques Ferlay, Freddie Bray, Ahmedin Jemal, (2018) International Patterns and Trends in Endometrial Cancer Incidence, 19782013, JNCI: Journal of the National Cancer Institute, Volume 110, Issue 4, Pages 354-361.

[3]Siegel, R. L., Miller, K. D., \& Jemal, A. (2016). Cancer statistics, 2016. CA: a cancer journal for clinicians, 66(1), 7-30.

[4]American Cancer Society. (2020) Cancer Facts and Figures 2020. Atlanta, Ga: American Cancer Society. https://www.cancer.org/content/dam/cancerorg/research/cancerfacts-and-statistics/annualcancer-facts-and-figures/2020/cancer-facts-andfigures2020.pdf 
[5]Lee, A.S. (2014) Glucose-regulated proteins in cancer: molecular mechanisms and therapeutic potential. Nat. Rev. Cancer 14, 263-276

[6]Bifulco, G., Miele, C., Di Jeso, B., Beguinot, F., Nappi, C., Di Carlo, C., ... \& Ulianich, L. (2012). Endoplasmic reticulum stress is activated in endometrial adenocarcinoma. Gynecologic oncology, 125(1), 220-225.

[7]Calì, G., Insabato, L., Conza, D., Bifulco, G., Parrillo, L., Mirra, P., ... \& Ulianich, L. (2014). GRP78 mediates cell growth and invasiveness in endometrial cancer. Journal of cellular physiology, 229(10), 1417-1426.

[8]Matsuo, K., Gray, M. J., Yang, D. Y., Srivastava, S. A., Tripathi, P. B., Sonoda, L. A., ... \& Lin, Y. G. (2013). The endoplasmic reticulum stress marker, glucose-regulated protein-78 (GRP78) in visceral adipocytes predicts endometrial cancer progression and patient survival. Gynecologic oncology, 128(3), 552-559.

[9]Guo, S., Xiao, Y., Li, D., Jiang, Q., Zhu, L., Lin, D., .. \& Lin, L. (2018). PGK1 and GRP78 overexpression correlates with clinical significance and poor prognosis in Chinese endometrial cancer patients. Oncotarget, 9(1), 680.

[10]Li, B., Cheng, X. L., Yang, Y. P., \& Li, Z. Q. (2013). GRP78 mediates radiation resistance of a stem celllike subpopulation within the MCF-7 breast cancer cell line. Oncology reports, 30(5), 2119-2126.

[11]Phillips, T. M., McBride, W. H., \& Pajonk, F. (2006). The response of CD24-/low/CD44+ breast cancerinitiating cells to radiation. Journal of the National Cancer Institute, 98(24), 1777-1785.

[12]Luvsandagva, B., Nakamura, K., Kitahara, Y., Aoki, H., Murata, T., Ikeda, S., \& Minegishi, T. (2012). GRP78 induced by estrogen plays a role in the chemosensitivity of endometrial cancer. Gynecologic oncology, 126(1), 132-139.

[13]Gray, M. J., Mhawech-Fauceglia, P., Yoo, E., Yang, W., Wu, E., Lee, A. S., \& Lin, Y. G. (2013). AKT inhibition mitigates GRP78 (glucose-regulated protein) expression and contribution to chemoresistance in endometrial cancers. International journal of cancer, 133(1), 2130 .

[14]Sommer, E. M., Dry, H., Cross, D., Guichard, S., Davies, B. R., \& Alessi, D. R. (2013). Elevated SGK1 predicts resistance of breast cancer cells to Akt inhibitors. Biochemical Journal, 452(3), 499508 .
[15]Bruhn, M. A., Pearson, R. B., Hannan, R. D., \& Sheppard, K. E. (2010). Second AKT: the rise of SGK in cancer signalling. Growth factors, 28(6), $394-408$

[16]Arteaga, M. F., Alvarez de la Rosa, D., Alvarez, J. A., \& Canessa, C. M. (2007). Multiple translational isoforms give functional specificity to serum-and glucocorticoid-induced kinase 1. Molecular biology of the cell, 18(6), 2072-2080.

[17]Talarico, C., Dattilo, V., D'Antona, L., Barone, A., Amodio, N., Belviso, S., ... \& Amato, R. (2016). SI113, a SGK1 inhibitor, potentiates the effects of radiotherapy, modulates the response to oxidative stress and induces cytotoxic autophagy in human glioblastoma multiforme cells. Oncotarget, 7(13), 15868 .

[18]Munakarmi, S., Shrestha, J., Shin, H. B., Lee, G. H., \& Jeong, Y. J. (2021). 3, 3'-Diindolylmethane Suppresses the Growth of Hepatocellular Carcinoma by Regulating Its Invasion, Migration, and ER Stress-Mediated Mitochondrial Apoptosis. Cells, 10(5), 1178.

[19]Verfaillie, T., Salazar, M., Velasco, G., \& Agostinis, P. (2010). Linking ER stress to autophagy: potential implications for cancer therapy. International journal of cell biology, 2010.

[20]Verfaillie, T., Garg, A. D., \& Agostinis, P. (2013). Targeting ER stress induced apoptosis and inflammation in cancer. Cancer letters, 332(2), 249264. 